\title{
The role of the acidic domain of $\alpha$-synuclein in amyloid fibril formation: A molecular dynamics study
}

SeongByeong Park ${ }^{\dagger}$, Jeseong Yoon ${ }^{\dagger}$, Soonmin Jang ${ }^{\ddagger}$, Kyunghee Lee ${ }^{\ddagger}$, and Seokmin Shin* ${ }^{\dagger}$ †Department of Chemistry, Seoul National University, Seoul 151-747, Republic of Korea $\neq$ Department of Chemistry, Sejong University, Seoul 143-747, Republic of Korea

\section{Supplementary material}

Figure S1. Representative configurations of the 4 clusters, obtained by performing a cluster analysis of trajectories of the simulations for the wild type ((a) to (d)) and P-to-A mutant ((e) to (h)) of the acidic domain (residues 101-140) of $\alpha$-synuclein at $300 \mathrm{~K}$ under the neutral condition. The numbers represent the fraction of each cluster, represented by the configuration.

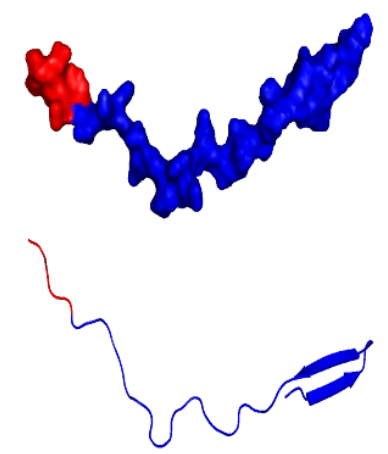

(a) 0.272
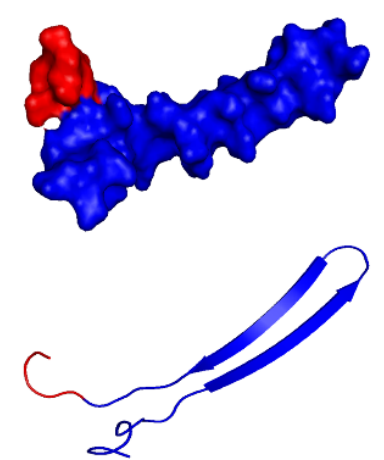

(e) 0.212

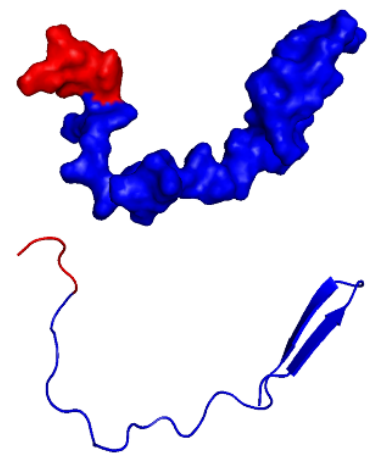

(b) 0.278

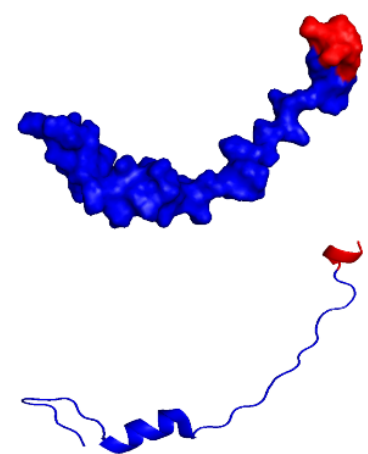

(f) 0.187

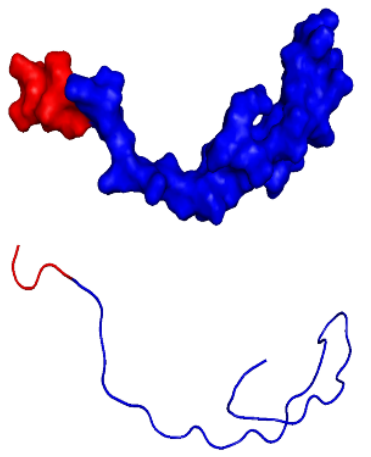

(c) 0.329

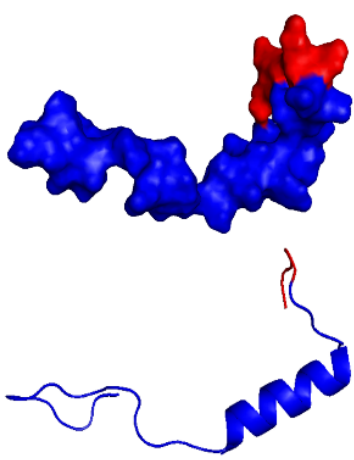

(g) 0.304

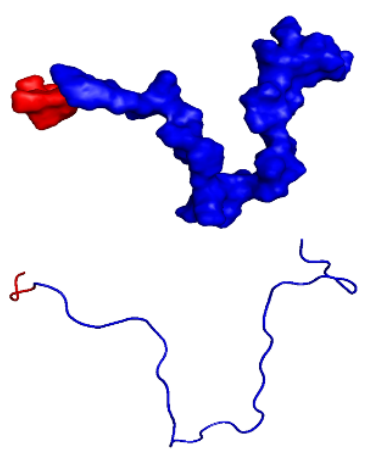

(d) 0.121

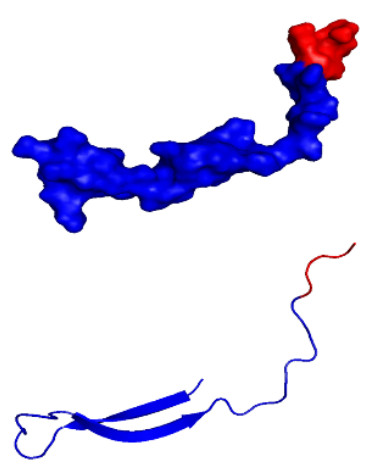

(h) 0.297 
Figure S2. Representative configurations of the 4 clusters, obtained by performing a cluster analysis of trajectories of the simulations for the wild type ((a) to (d)) and P-to-A mutant ((e) to (h)) of the acidic domain (residues 101-140) of $\alpha$-synuclein at $300 \mathrm{~K}$ under the acidic condition. The numbers represent the fraction of each cluster, represented by the configuration.

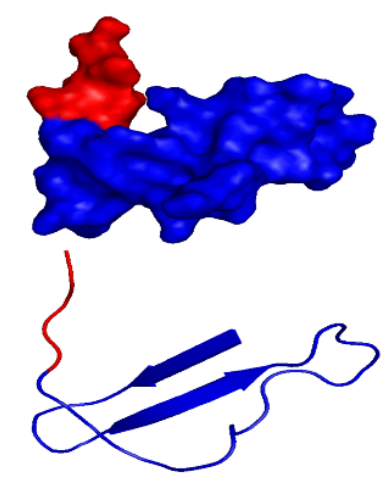

(a) 0.196

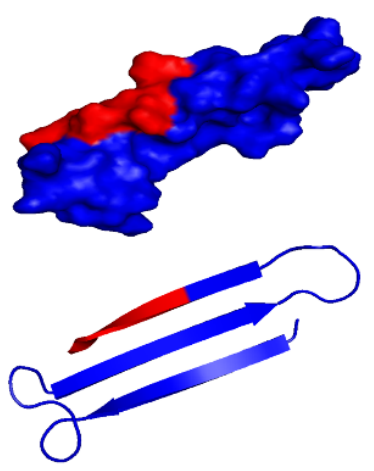

(e) 0.394

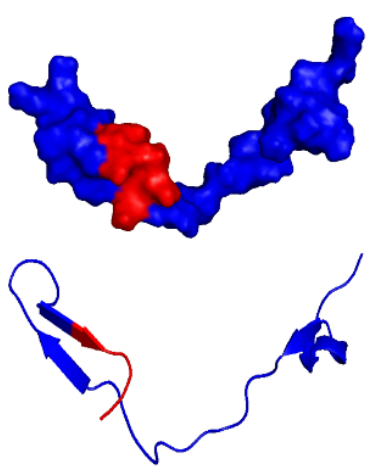

(b) 0.304

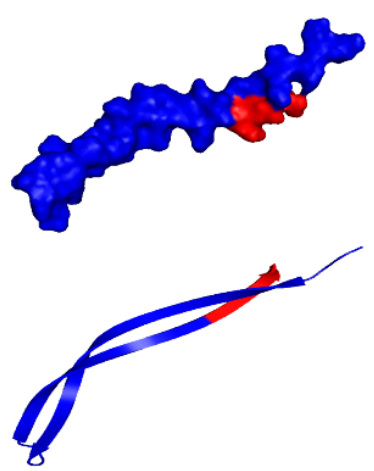

(f) 0.299

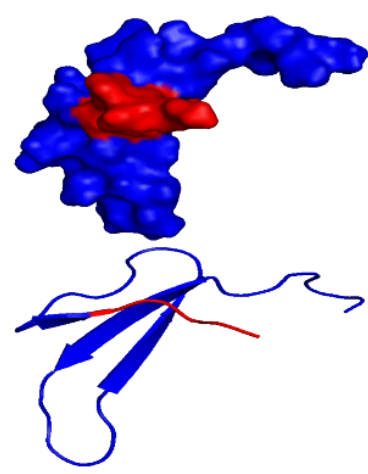

(c) 0.306

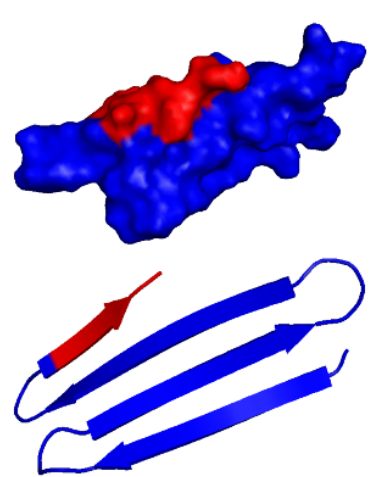

(g) 0.306

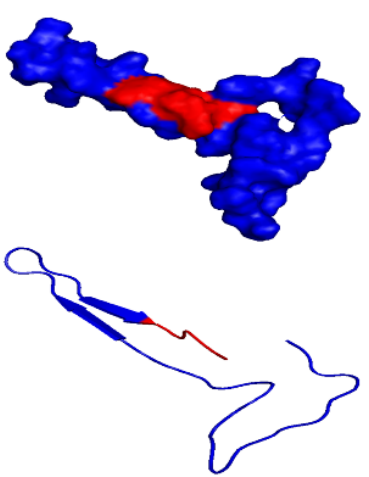

(d) 0.194

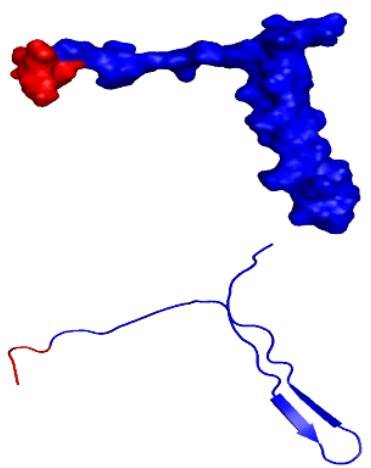

(h) 0.001 
Figure S3. Representative configurations of the 4 clusters, obtained by performing a cluster analysis of trajectories of the simulations for the wild type ((a) to (d)) and P-to-A mutant ((e) to (h)) of the combined sequence (residues 61-140) of the NAC domain (blue) and the acidic region (red) of $\alpha$ synuclein at $300 \mathrm{~K}$ under the neutral condition. The numbers represent the fraction of each cluster, represented by the configuration.
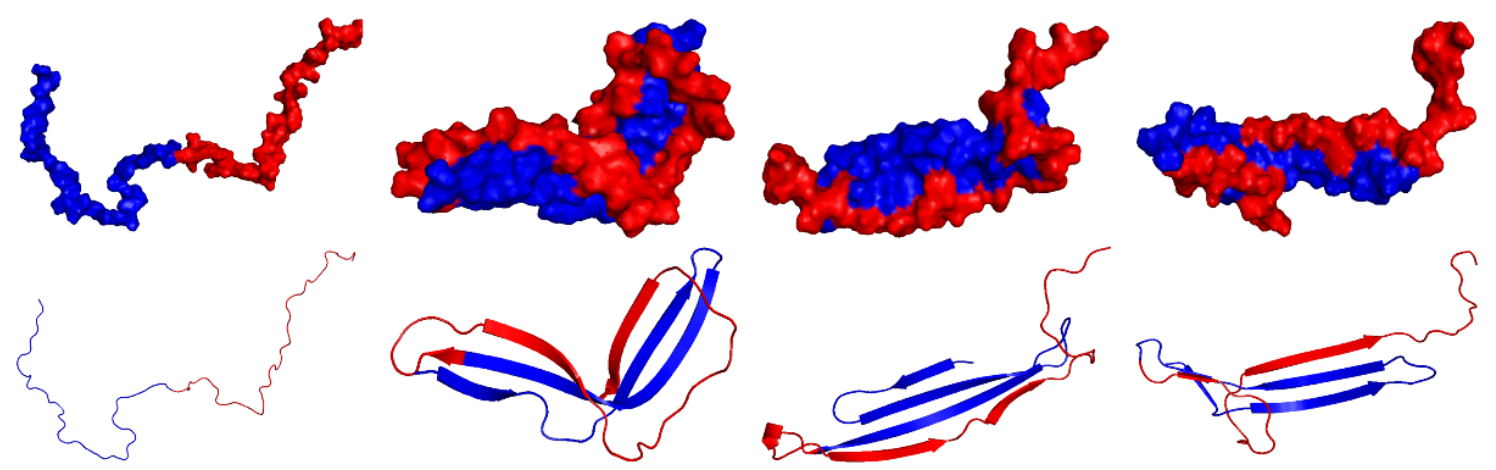

(a) 0.001

(b) 0.720

(c) 0.237

(d) 0.042
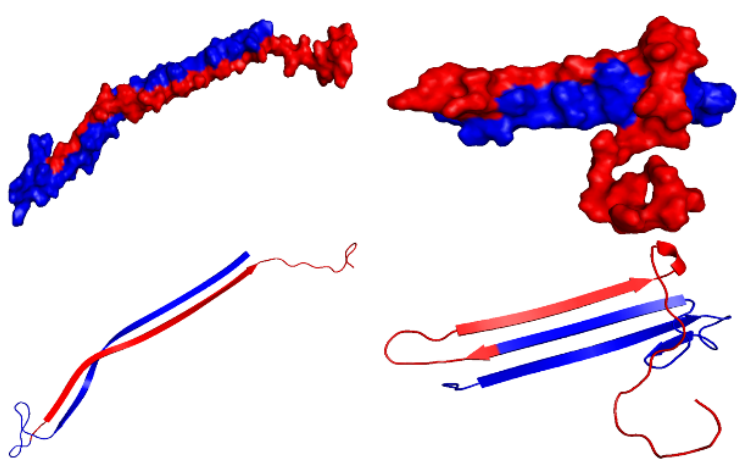

(e) 0.001

(f) 0.402

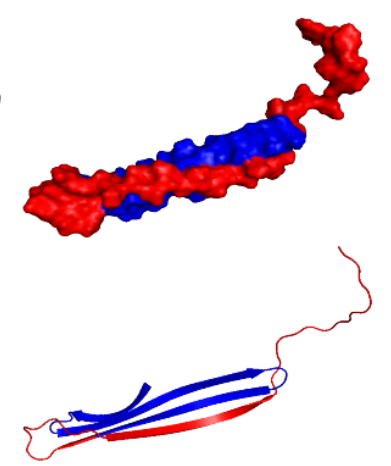

(g) 0.337

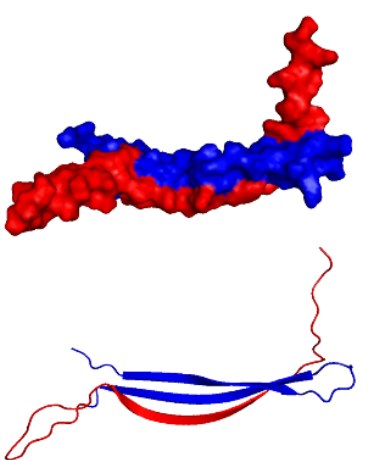

(h) 0.260 
Figure S4. Representative configurations of the 4 clusters, obtained by performing a cluster analysis of trajectories of the simulations for the wild type ((a) to (d)) and P-to-A mutant ((e) to (h)) of the combined sequence (residues 61-140) of the NAC domain (blue) and the acidic region (red) of $\alpha$ synuclein at $300 \mathrm{~K}$ under the acidic condition. The numbers represent the fraction of each cluster, represented by the configuration.

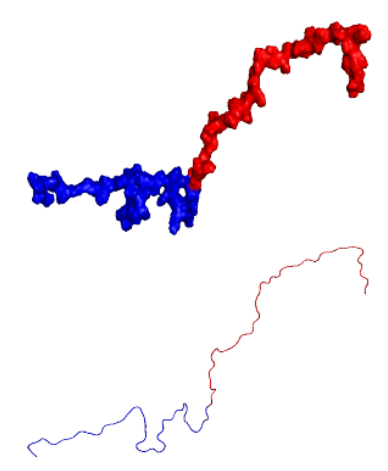

(a) 0.001
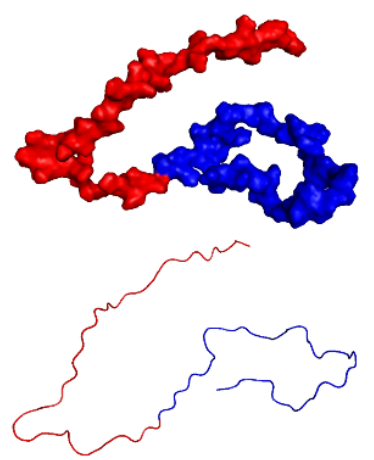

(e) 0.001

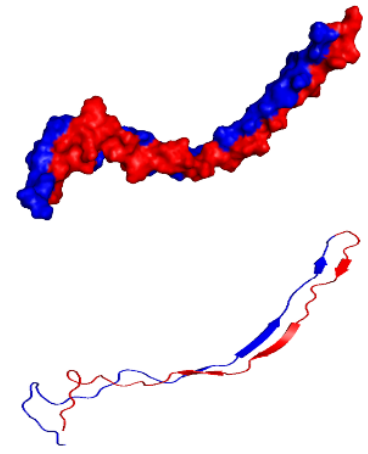

(b) 0.154
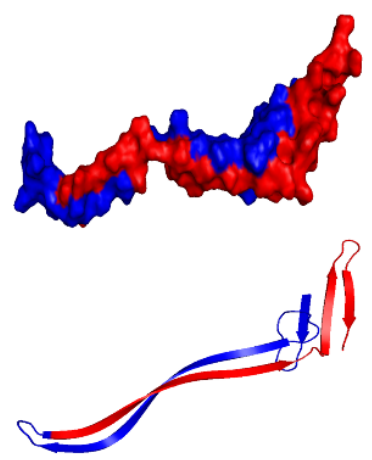

(f) 0.214

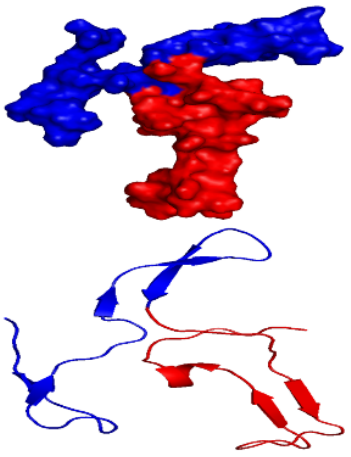

(c) 0.527
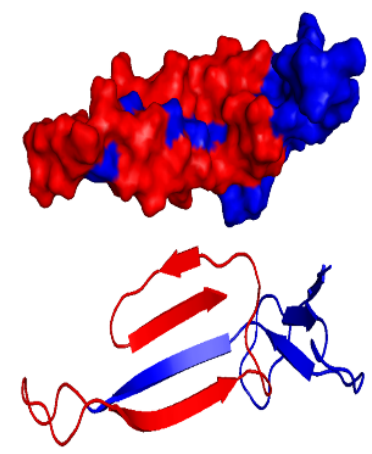

(g) 0.783
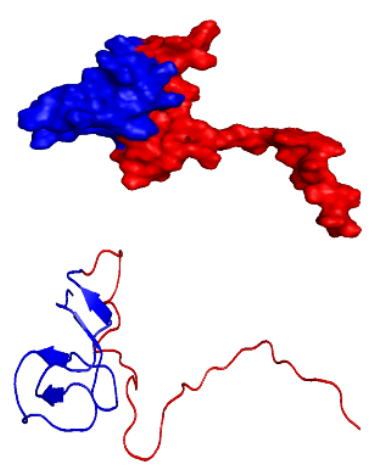

(d) 0.318

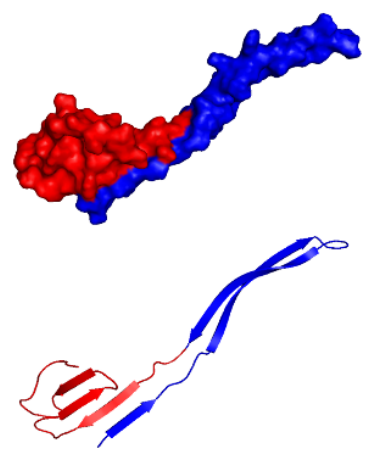

(h) 0.002 
Figure S5. Contact maps for the wild type of the combined sequence (residues 61-140) of NAC domain and the acidic region of $\alpha$-synuclein at $300 \mathrm{~K}$ for (a) WT at neutral condition, (b) P-to-A mutant at neutral condition, (c) WT at acidic condition, and (d) P-to-A mutant at acidic condition.

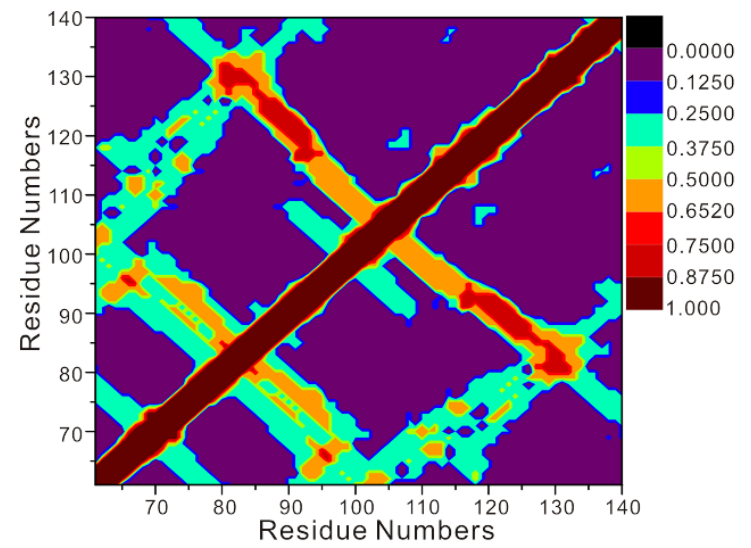

(a)

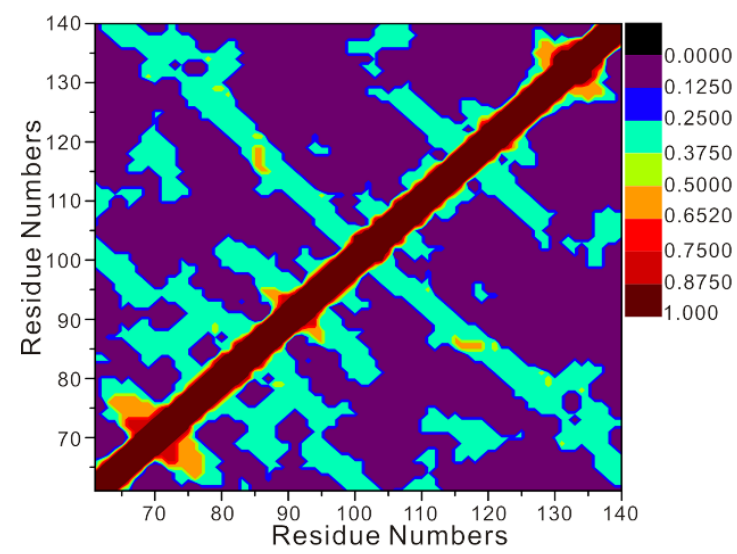

(c)

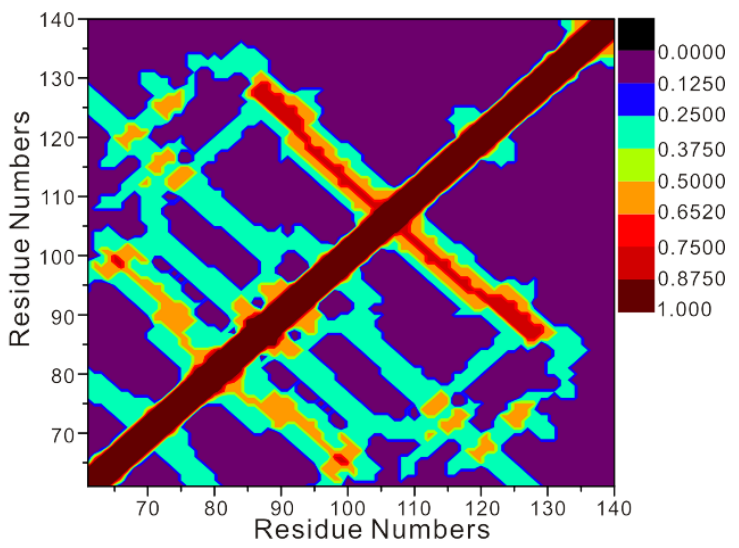

(b)

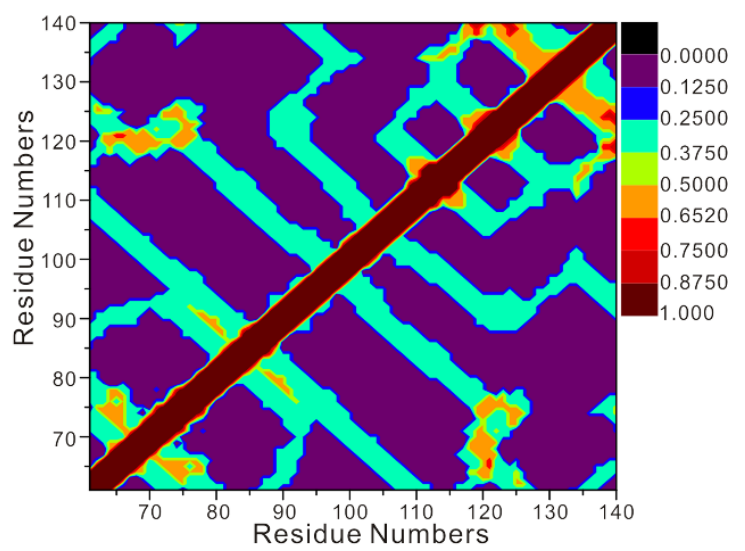

(d) 
Table S1. The ratio of the solvent accessible surface area (SASA) of $\alpha$-synuclein at different conditions to the reference SASA for the WT at neutral condition. The averaged SASAs for both the combined region of the NAC and acidic domain and the NAC domain alone are compared.

\begin{tabular}{lcc}
\hline SASA/SASA(Ref) & NAC + Acidic & NAC \\
\hline WT at neutral & 1.000 & 1.000 \\
P-to-A at neutral & 1.076 & 1.162 \\
WT at acidic & 1.086 & 1.283 \\
P-to-A at acidic & 1.014 & 1.252 \\
\hline
\end{tabular}

